\title{
Vom Bürgerrecht auf Erden und im Himmel - Religiöse Bildung in weltzivilgesellschaftlicher Perspektive
}

Schlag, Thomas

Posted at the Zurich Open Repository and Archive, University of Zurich ZORA URL: https://doi.org/10.5167/uzh-69353

Journal Article

Published Version

Originally published at:

Schlag, Thomas (2012). Vom Bürgerrecht auf Erden und im Himmel - Religiöse Bildung in weltzivilgesellschaftlicher Perspektive. Zeitschrift für Pädagogik und Theologie, 64(3):238-250. 
deutlich, wie das wechselseitige Verstehen für den respektvollen Umgang miteinander wesentlich ist.

d) Grenzen durch fremde Situation überschreiten: Kleingruppen recherchieren im Internet verschiedene Gemeindesituationen in eher problematischen Regionen (z. B. Osteuropa, Südamerika, Afrika) und sammeln Informationen durch Mailkommunikation in vermutlich englischer Sprache. Die Gemeinsamkeiten und Unterschiede mit der eigenen Gemeinde werden dargestellt. Im Sinn des ökumenischen Imperativs von Joh 17, 21 „Auf dass sie alle eins seien“ versuchen die Kleingruppen, Kontakt zu der fremden Gemeinde zu halten und Entwicklungen zu verstehen. Unterstützungsmöglichkeiten können dadurch entstehen.

Es wurde deutlich, dass in einer multikulturellen Schule die Sichtweise auf die individuelle Transkulturalität die Chance in sich birgt, in Prozessen des Lernens, differenzhermeneutisch das Eigene im Fremden zu entdecken. Dies überwindet die Xenophobie, die Angst vor dem Anderen und die Angst vereinnahmt zu werden, da die Differenzen bestehen bleiben und akzeptiert werden. Durch ökumenisches Lernen als Lernprozess des Individuums in der Interaktion mit seiner Umwelt unter Berücksichtigung der subjektiven kulturellen Werte und der vorhandenen (trans-)kulturellen Besonderheiten erwerben die Heranwachsenden ihre Identität. Mit Martin Buber ${ }^{25}$ verkürzt gesprochen: „Ich werde am Du!“

Thomas Schlag

\section{Vom Bürgerrecht auf Erden und im Himmel - Religiöse Bildung in weltzivilgesellschaftlicher Perspektive}

\section{Erfahrungen}

Auf dem Tisch liegt ausgebreitet eine grosse topografische Karte des Irak im Maßstab 1: 50 000, über die sich die Jugendlichen konzentriert beugen. Jeder der zwölf Realschüler hat eine Anzahl von unterschiedlich farbigen Spielfiguren und ein je eigenes schriftliches Argumentationsarsenal sowie verschiedene Ressourcenkarten vor sich: Der eine soll die Interessen der Kurden, der andere die der Schiiten, der dritte die der Sunniten vertreten. Dann gibt es da die alliierten Streitkräfte, die Vertreter der Vereinten Nationen und der diplomatischen Vertretungen der EU-Länder, die karitativen Hilfswerke, Industrie und Ölgesellschaften, die Manager der Tourismusbranche sowie schließlich die Vertretung der religiösen Minderheit der Christen. Jeder soll sich mit seinen Spielfiguren - natürlich hat jeder unterschiedlich viele Figuren - seine Position

${ }^{25}$ Vgl. Martin Buber, Das dialogische Prinzip. Heidelberg ${ }^{4} 1979,15$. 
im Land suchen und möglichst überzeugend seine eigene Rolle vertreten. Im Planspiel soll es darum gehen, möglichst verlässliche Kooperationen zu bilden, um am Ende einen gemeinsamen Friedensvertrag für die weitere Zukunft des Landes abzuschließen. Pädagogisches Ziel des Planspiels - so durchgeführt im Rahmen einer Jugend-Tagung an einer Evangelischen Akademie - sollte es sein, zum einen für die Komplexität der Situation zu sensibilisieren, zum anderen die Konsequenzen spürbar zu machen, die sich bei kompromisslosem Verharren auf den eigenen Interessen notwendigerweise einstellen müssen und schließlich die Frage nach alternativen zivilisierten und zivilisierenden Maßnahmen zu stellen.

In der Durchführung kommt es nun zu anfangs recht offenen, dann immer langwierigeren Verhandlungen, die zunehmend aggressiver geführt wurden, schliesslich eskalieren und in ein imaginiertes Gewaltszenario münden. Auch die religiösen Unterschiede und die unterschiedlichen ethnisch-kulturellen Hintergründe tragen erheblich zur Befeuerung der Gesamtkonstellation bei. All dies führt bei den Jugendlichen irgendwann zur frustrierenden Einsicht, dass am Ende - zumindest wenn die Positionen so verhärtet bleiben - alle Verlierer sein werden. In der Auswertung zeigt sich bei den Jugendlichen einerseits viel Verständnis für die unterschiedlichen Interessen, aber auch erheblicher Ärger über die Ignoranz und Blindheit aller beteiligten Akteure und deren kompromisslosen Durchsetzungswillen. Eine weitere Eskalation, die dann ja auch faktisch erlebbar wurde, scheint kaum mehr zu vermeiden zu sein.

In der Beobachtung und Schlussauswertung des Ganzen zeigt sich auf eindrückliche Weise, dass dieses Szenario für die Jugendlichen nicht einfach ein friedlich schiedliches Planspiel des herrschaftsfreien Dialogs darstellt, das sich irgendwo in einem keimfreien Völkerparlament abspielt, sondern von ihnen selbst eben als stellvertretende Akteure gleichsam mit Haut und Haaren miterlebt wird. Niemand blieb insbesondere von den immer wieder scheiternden Verhandlungen unberührt - unmittelbar nachvollziehbar wurde, dass es in dieser konkreten weltpolitischen Konfliktlage tatsächlich um die Sicherung der Existenz und des Überlebens von Menschen und damit um nicht weniger als eine Frage von Leben und Tod geht. Dass die individuelle Rollenübernahme diese Sensibilität beförderte, wurde sowohl für die Jugendlichen wie die das Planspiel leitenden Erwachsenen unmittelbar miterlebbar.

Die geschilderte Erfahrung wirft für die Aufgabe einer religiösen Bildung und Bildungsverantwortung in weltzivilgesellschaftlicher Perspektive eine Reihe von Grundfragen auf, die im Folgenden näher bedacht werden sollen:

\section{Zum Begriff der Weltzivilgesellschaft}

Der Begriff einer Weltzivilgesellschaft ist mindestens bisher explizit wenig gebräuchlich. Interessantweise war es der katholische Theologe Leonardo Boff, der im Zusammenhang des seit 2001 regelmässig in Porto Alegre stattfindenden Weltsozialforums davon sprach, dass hier „allmählich eine Weltzivilgesellschaft entsteht“. ${ }^{1}$ Ein Jahr spä-

${ }^{1}$ Der Standard, vom 30.1.2005 [http://derstandard.at/1933969: Zugriff am 11.1.2012]. 
ter fand in Genf unter Beteiligung der Vereinten Nationen und vieler Nichtregierungsorganisationen das erste so genannte „World Civil Society Forum“ statt, dessen Ziel in der Schaffung von „space for dialogue on the relation between the different stakeholders of the international scene, such as civil society organizations, international organizations, governments and the private sector ${ }^{\text {“2 }}$ bestimmt wurde. Auch wenn sich in den vergangenen zwei Jahrzehnten viele solcher internationalen zivilgesellschaftlichen Dialoge ergeben haben, hat sich diese zugegebenenermassen sperrige Begrifflichkeit bisher nicht etabliert. Der seit den 1990er Jahren anzutreffende Neologismus „Global Civil Society“ wird aber offenbar langsam „fashionable“.3 Dabei gilt nun zugleich, auch dies mag die zögerliche Durchsetzung erklären, dass der Begriffsgebrauch selbst, der von stark analytisch-deskriptiven bis hin zu normativ-idealistischen Verwendungen führt, einigermaßen unüberschaubar ist.

Viel eher ist, wenn diese neuen politischen Kommunikations-, Verantwortungs- und Entscheidungsstrukturen in der globalen und risikobehafteten Moderne sowie die damit verbundenen Bildungsprozesse thematisiert werden, von „der Weltgesellschaft“ die Rede. Hier stellt für den deutschsprachigen Zusammenhang N. Luhmanns Konzeption von "Weltgesellschaft“ als Sozialsystem, das alle anderen Sozialsysteme in sich einschließt, die entscheidende Referenz dar. ${ }^{4}$

Als weiteres wesentliches - und auch pädagogisch relevantes - Charakteristikum für die Rede von einer Weltgesellschaft wird dabei die Tatsache einer gemeinsamen, alle nationalen Grenzen überschreitenden Interdependenz- und Kommunikationsebene genannt. Die Rede ist in diesem Zusammenhang vom Phänomen einer welterschließenden Weltkommunikation, die sich durch prinzipielle Erreichbarkeit und das Phänomen von „small worlds“ bzw. „networks“ auszeichnen. Weltgesellschaft kann somit als „Diversität oder Pluralität von small worlds“ ${ }^{5}$ beschrieben werden. Zudem zeichnet sich Weltgesellschaft durch eine gemeinsame Verstehens- und Anschlussfähigkeit aus, die gerade durch eine dekontextualisierte Symbolik zustande kommt. Diese kann ihrerseits dann besonders gut kommuniziert werden, wenn an die Stelle „echter“ Interaktion symbolisch generalisierte mediale Kommunikation tritt, die zugleich Raum und Zeit domestiziert und kontrolliert. ${ }^{6}$ All diese Charakteristika stellen gleichsam wesentliche Elemente einer weltgesellschaftsbewussten religionsdidaktischen Reflexion dar,

\footnotetext{
${ }^{2}$ http://www.worldsociety.org/pages/1/en/presfor.htm [Zugriff am 11.1.2012]

3 J. Keane, Global Civil Society? Cambridge 2003, 1; aus eher aktivistischer Perspektive vgl. M. Kaldor, World Civil Society. An Answer to War. Oxford 2003.

${ }^{4}$ Der Luhmannsche Begriff der Weltgesellschaft integriert dabei zwei Perspektiven: „Einerseits heißt dies, dass es auf dem Erdball und sogar in der gesamten kommunikativ erreichbaren Welt nur eine Gesellschaft geben kann. Das ist die strukturelle und die operative Seite des Begriffs. Zugleich soll der Ausdruck Weltgesellschaft aber auch sagen, dass jede Gesellschaft (und im Rückblick gesehen: auch die Gesellschaften der Tradition) eine Welt konstruiert und das Paradox des Weltbeobachters dadurch auflöst.“, N. Luhmann, Die Gesellschaft der Gesellschaft. Frankfurt/M. 1997, 72.

${ }^{5}$ Vgl. R. Stichweh, Setzt die „Weltgesellschaft“ auf „Weltkommunikation“?, in: M. Jaeckel/F. Haase (Hg.), In medias res. Herausforderung Informationsgesellschaft. München 2005, 175-190, hier 181.

${ }^{6}$ Vgl. a. a. 0., 187.
} 
wovon noch die Rede sein wird. Zuvor aber ist von den genannten Aspekten aus die hier favorisierte Rede von der Weltzivilgesellschaft selbst noch zu erläutern.

Der Begriff von der Weltzivilgesellschaft eröffnet, so die These, gegenüber dem Begriff der Weltgesellschaft über die strukturellen Fragen hinaus einen genaueren Blick auf die eigentlichen Kommunikationsakteure bzw. die notwendigen Bedingungen einer gelingenden Kommunikation über die Zukunftsfragen der globalen Moderne - genannt sei hier etwa nur die Schaffung neuer, eben personbezogener Netzwerke gerade als Voraussetzung gelingender Bildung ${ }^{7}$ : Im Sinn eines Idealtypus kann von einer Weltzivilgesellschaft die Rede sein „as an unfinished project that consists of sometimes thick, sometimes thinly stretched networks, pyramids and hub-andspoke-clusters of socio-economic institutions and actors who organise themselves across borders, with the deliberate aim of drawing the world together in new ways. These non-governmental institutions actors tend to pluralise power and to problematise violence; consequently, their peaceful or 'civil' effects are felt everywhere, here and there, far and wide, to and from local areas, through wider regions, to the planetary level itself". ${ }^{8}$ Oder wie es vor kurzem von Seiten der London School of Economics formuliert wurde: "Global Civil Society is about understanding globalisation 'from below', from the perspective of ordinary people. We see the concept as describing an emerging reality of global civic action and connectedness".

Dass sich in den vergangenen etwa zwei Jahrzehnten tatsächlich ganz neue weltgesellschaftliche Formen einer solchen personbezogenen politischen Artikulation, Vernetzung und Verbreiterung bestehender Verantwortungs- und Entscheidungskompetenzen etabliert haben und diese Ausweitung durch die digitalen Möglichkeiten weiter anwächst, ist offensichtlich. Kurz gesagt: In pädagogischer Hinsicht wird die Vorbildhaftigkeit etwa von NGO's erst dann wirklich deutlich, wenn sich auf Seiten der Lernenden damit persönliche Gesichter und Geschichten verbinden lassen und diesen Gesichtern und Geschichten eine eigene persönliche Bedeutung beigemessen werden kann.

Hiermit verbindet sich auch ein neuer Blick auf Handlungs- und auch Einflussmöglichkeiten innerhalb des politischen Sektors im Sinn der Ausweitung der bisherigen gouvernementalen Politiksteuerung auf die unterschiedlichsten zivilgesellschaftlichen Akteure $^{10}$, wie der erhebliche Anstieg entsprechender Aktivitäten in den letzten Jahren ebenso eindrücklich aufzeigen wie die jüngsten Entwicklungen etwa der arabischen Revolutionen. Angesichts dieser noch kaum abschätzbaren Bürgerbewegungen, der umfassenden kapitalistischen Kolonisierung der gegenwärtigen globalen Lebenswelten sowie der bewussten und unbewussten Ausschlüsse aus den globalen Kommunika-

${ }^{7}$ Vgl. L. Böhnisch/W. Schröer, Politische Pädagogik. Eine problemorientierte Einführung. Weinheim/München 2007, v. a. 272-283.

8 J. Keane, Global Civil Society? Cambridge 2003, 8.

${ }^{9} \mathrm{http}: / /$ www2.lse.ac.uk/globalGovernance/research/globalCivilSociety/home.aspx [Zugriff: 20.1.2012]

10 Vgl. dazu D. Messner, Regieren in der Weltgesellschaft - Probleme und Perspektiven der Global Governance, in: W. Sander/A. Scheunpflug (Hg.), Politische Bildung in der Weltgesellschaft. Herausforderungen, Positionen, Kontroversen. Bonn 2011, 34-50. 
tionsprozessen erscheint jedenfalls der Begriff der Weltgesellschaft ohnehin als beinahe zu harmlos.

Wird nun innerhalb dieses Beitrags folglich von einer weltzivilgesellschaftlichen Perspektive gesprochen und diese auf Fragen religiöser Bildung hin bedacht, so soll damit gerade dem Aspekt des „Zivilen“ im Sinn einer kompetenten Kommunikations-, Verantwortungs- und Entscheidungsteilhabe seiner Bürgerinnen und Bürger eine stärkere explizite Erkennbarkeit gegeben werden und damit die Ebene der persönlichen zivilgesellschaftlichen Handlungsspielräume angesichts der globalen Dynamiken ausdrücklich in den Blick kommen. ${ }^{11}$ Mir erscheint diese Rede vom „Zivilen“ zugleich auch ein noch offenerer und universalisierbarer, weil weniger westlich-aufgeklärt vorgeprägter, Begriff als der einer weltbürgerlichen Erziehung zu sein, von der ja ebenfalls gegenwärtig intensiv die Rede ist ${ }^{12}$ - ganz abgesehen von der Frage, ob man in anthropologischer Hinsicht überhaupt von einer menschlichen Bereitschaft zum Weltbürger-Sein sprechen kann. ${ }^{13}$

Durch den Begriff des „Zivilen“ - sowohl im Sinn der zivilisierten wie zivilisierenden Formen eines global orientierten, notwendigen Handelns - können dann auch die konkreten Bildungsherausforderungen und -prozesse profilierter in den Blick genommen werden.

Schliesslich können durch eine Rede von einer Weltzivilgesellschaft aber gerade von dieser persönlichen Ebene aus dann auch die konkreten Nichtregierungsorganisationen in ihrem Anspruch, zur Entwicklung lokaler, regionaler und nationaler Zivilgesellschaft(en) beizutragen, angemessener und konkreter zum Vorschein kommen. Dies ist insofern für eine religiöse Bildung von Belang, als im vorliegenden Zusammenhang sowohl Schule wie Kirche als zivilgesellschaftlich relevante intermediäre Institutionen in das Blickfeld rücken sollen. Auch hier gilt wiederum, dass diese nur dann in ihrer Bedeutsamkeit verstanden werden können, wenn sich dies mit einer erheblichen personbezogenen Kommunikationsdynamik verbindet. Die globalen Interdependenzen und Entgrenzungen bringen folglich ganz neue pädagogische Herausforderungen und Chancen bis hin zu neuen Konzepten eines „Aktiven-Politik-Lernens“14 mit sich.

Gegenüber einer eher anonymen organisationssoziologischen Betrachtungsweise, die sich mit dem Begriff der Weltgesellschaft verbinden mag, rücken folglich durch die Rede von der Weltzivilgesellschaft stärker tatsächlich die Herausforderungen für jeden Einzelnen und einzelne Gruppen - und damit überhaupt Personen, Gesichter,

\footnotetext{
${ }^{11}$ Vgl. M.A. Peters/A. Britton/H. Blee (Eds.), Global Citizenship Education. Philosophy, Theory and Pedagogy. Rotterdam 2008.

${ }^{12}$ Vgl. dazu etwa K. Seitz, Bildung in der Weltgesellschaft. Gesellschaftstheoretische Grundlagen Globalen Lernens. Frankfurt/M. 2002.

13 Vgl. dazu A.K. Treml, Globalisierung als pädagogische Herausforderung. Möglichkeiten und Grenzen einer weltbürgerlichen Erziehung, in: W. Sander/A. Scheunpflug (Hg.), Politische Bildung in der Weltgesellschaft. Herausforderungen, Positionen, Kontroversen. Bonn 2011, 190-203, hier 195.

14 Vgl. B. Widmaier, Mehr Demokratie und mehr Politik wagen. Partizipation als Ziel der Politischen Bildung, in: D. Lange (Hg.), Entgrenzungen. Gesellschaftlicher Wandel und Politische Bildung. Schwalbach/Ts. 2011, 269-274, hier 273.
} 
deren Schicksale und Geschichten - ebenso wie die faktische Weltverflochtenheit und die Notwendigkeit funktionierender intermediärer Institutionen in den Blick.

\section{Beteiligungsperspektiven religiöser Bildung}

Dass die Phänomene der Weltzivilgesellschaft „ein Fall für“ religiöse Bildung sind, lässt sich in unterschiedlicher Hinsicht begründen. Zum einen gehört es zum allgemein bildenden Auftrag religiöser Bildung, sich mit den politischen und gesellschaftlichen Entwicklungen der jeweiligen Gegenwart aktiv und bewusst auseinander zu setzen. Sie ist es ihrer inhaltlichen und institutionellen Verortung ebenso wie der eigenen Bildungsklientel, sei es im Zusammenhang formaler, non-formaler oder informeller Bildung, schuldig, sachlich begründete Deutungsperspektiven der Entwicklungen der globalen Moderne anzubieten. Die Weckung des Bewusstseins für die zivilgesellschaftlichen Gestaltungsmöglichkeiten demokratischen Lebens soll und kann von Seiten schulischer und kirchlicher religiöser Bildung aus nicht als sachfremdes Feld verstanden werden, das man womöglich ganz und gar den säkularen politischen Bildungsträgern, den entsprechenden Schulfächern Gemeinschaftskunde, Geschichte oder Politik und damit in fachdidaktischer Hinsicht ausschließlich der Politik- und Geschichtsdidaktik überlassen sollte. ${ }^{15}$

Zudem ist daran zu erinnern, dass gesellschaftliche Verantwortungsträger, in welchem späteren Beruf auch immer, mehr denn je auch über eine hohe Wahrnehmungsund Deutungskompetenz in religiösen Fragen verfügen müssen. Gerade Kosmopoliten sollten um die religiösen Bezüge wissen, in denen sie sich vermeintlich weltgewandt bewegen. ${ }^{16}$ Wobei zugleich gilt, dass es sich dabei eben nicht nur um ein ökonomistisch-verkaufsrelevantes Erlernen von Höflichkeitsstandards anderer Kulturen und Länder handeln kann, wie dies in den entsprechenden Managementseminaren gelehrt wird.

Aber auch aus institutionellen Gründen legt sich eine intensive Beschäftigung mit diesen globalen Prozessen nahe, da gerade Kirchen, religiöse Gemeinschaften und religiös motivierte Gruppierungen einflussreiche Größen in den je regionalen politischen und zivilgesellschaftlichen Verhältnissen darstellen. Dies gilt nun sowohl in positiver wie in problematischer Hinsicht:

Zum einen sind es oftmals überhaupt die religiösen und kirchlichen Gruppen, die eine zivilgesellschaftliche Dynamik vor Ort durch ihr Engagement und ihre Kommunikationsformen auslösen oder befördern. Oftmals sind diese für die benachteiligten Bevölkerungsgruppen vor Ort wichtige Anlauf- und Hilfsstellen und auch für diplomatische Vertretungen wichtige Informationsmedien über die tatsächlichen Zustände etwa in einer bestimmten Konfliktregion. Ein ziviles, zivilisiertes und zivilisierendes Engagement kann gerade von religiöser Seite aus durch eine spezifische Menschenrechts-,

\footnotetext{
${ }^{15}$ Vgl. T. Schlag, Horizonte demokratischer Bildung. Evangelische Religionspädagogik in politischer Perspektive. Freiburg i.Br./Basel/Wien 2010.

${ }^{16}$ Vgl. S. Prothero, Religious Literacy. What every American needs to know - and doesn't. New York 2007.
} 
Friedens- und Versöhnungspolitik und ein überparteiliches Interesse manifest werden.

Zum anderen sind es ja gerade religiöse Kräfte, die im Einzelfall inmitten global relevanter Konflikte mindestens mit präsent sind, um es einmal zurückhaltend zu formulieren. Bestimmte national-nationalistische Exklusionstendenzen sind jedenfalls offenbar kein Privileg der entsprechenden politischen Parteien, sondern können sich auch in einzelnen Religionsgemeinschaften und Kirchen - und dies mitten in Europa - niederschlagen.

Religiöse Bildung kann also angesichts der unterschiedlichsten weltzivilgesellschaftlichen Zusammenhänge dazu dienen, bestimmte Konflikte und Konfliktlinien überhaupt besser einordnen zu können, konkret etwa Kriterien für eine bessere Differenzierung zwischen kulturellen und religiösen Hintergründen gewinnen und dabei zugleich auch Verständnis für die jeweiligen religiös artikulierten Interessen entwickeln zu können.

Schließlich ist daran zu erinnern, dass das so genannte Phänomen der Globalisierung $^{17}$ in besonderer Weise auf dem Sektor religiöser Identität und eines entsprechenden gruppenbezogenen Urteilens und Handelns relevant ist. Gerade in religiösen Fragen können sich globale und lokale Bezüge und zugleich institutionelle und hochpersönliche Faktoren - im Sinn eigener Identität und Zugehörigkeit - sowohl auf problematische wie auch auf hoch kreative Weise miteinander verbinden. ${ }^{18}$

\section{Die weltzivilgesellschaftliche Perspektive im Kontext religiöser Bildung}

Die religiöse Bildung in Schule und Kirche hat sich spätestens seit den frühen 70er Jahren des vergangenen Jahrhunderts intensiv mit unterschiedlichen globalen Entwicklungen auseinandergesetzt und dabei sowohl Perspektiven eines ökumenischen wie globalen Lernens stark entwickelt. ${ }^{19}$ Ohne hier auf die Einzelheiten einzugehen, kann davon gesprochen werden, dass gerade der schulische Religionsunterricht als eines der ersten Unterrichtsfächer sehr frühzeitig auch die ökonomischen und dann auch die ökologischen Entwicklungen der damals so genannten 3. Welt in den Blick genommen und die Kinder und Jugendlichen für die Fragen weltweiter Gerechtigkeit und Ungerechtigkeit sensibilisiert hat.

Dass dabei in den entsprechenden Lehrmitteln lange Zeit eine gewisse Faszination für ferne Exotik mitschwang und sich mindestens unterschwellig eine Art kolonialer Hierarchie zwischen erster und dritter Welt mit den entsprechend zwiespältigen Empathieerklärungen für die Entrechteten dieser Welt abbildete, ist gleichwohl nicht von der Hand zu weisen - wie sich im Umkehrschluss schon an einer entsprechenden

\footnotetext{
17 Vgl. R. Robertson, Glokalisierung: Homogenität und Heterogenität in Raum und Zeit, in: U. Beck (Hg.), Perspektiven der Weltgesellschaft, Frankfurt/M. 1998, 192-220.

${ }^{18}$ Eine eindrückliche vergleichende Feldstudie zum globalen Charakter lokaler religiöser Institutionen und deren weltzivilgesellschaftlichen Gestaltungsanspruch findet sich bei M. Juergensmeyer, The Church, the Mosque and Global Civil Society, in: Global Civil Society Yearbook 2006/07, 144-159.

${ }^{19}$ Siehe die Beiträge von Simojoki und Scheunpflug in diesem Band.
} 
Denkschrift der EKD aus dem Jahr 1973 zeigt, in der bewusst eine Erziehung zur Entwicklungsverantwortung gefordert wird, und sowohl alle Überlegenheitsgefühle vermieden wie auch die politischen, wirtschaftlichen und sozialen Ursachen von Armut mit in den Blick genommen werden müssten. ${ }^{20}$ Zudem zeigt sich durch die Lehrmittel des letzten halben Jahrhunderts hindurch, dass die Thematisierung von Weltfragen immer auch in erheblicher Weise vom jeweiligen religionsdidaktischen Gesamtanspruch geprägt war, was nicht selten zu problematischen Funktionalisierungen - vor allem im Zusammenhang eines problemorientierten Religionsunterrichts in seiner politisierenden Spielart - führte.

Auch hat die ausführliche Behandlung religiös und prophetisch motivierter Weltbürger wie Albert Schweitzer, Mahatma Gandhi, Martin Luther King oder Rigoberta Menchú zwar ganze Generationen von Jugendlichen etwa für die Fragen ausbeuterischer Kolonisierung, Rassendiskriminierung und des zivilen Ungehorsams sensibilisiert - die Frage ist allerdings, ob dabei die je spezifischen kulturellen Kontexte dieser globalen Säulenheiligen durchweg mitbedacht wurden oder ob nicht die Leistungen dieser Personen allzu schnell in ihrer Vorbildfunktion auf die mitteleuropäischen Verhältnisse transponiert wurden.

Ob die pädagogischen Effekte grösser wurden, als man dann etwa seit den 90er Jahren immer stärker an die persönliche Verantwortung der Kinder und Jugendlichen für globale Ungerechtigkeiten appellierte, muss mindestens offen bleiben. Jedenfalls ist mitnichten klar, welche Einsichten und auch Widerstände man bei Kindern und Jugendlichen durch die einschlägigen Berichte über fernöstliche Turnschuh-, Fussballund Computerfabriken erzeugte - dass die Erregung individuellen Schulbewusstseins jedenfalls selbst von nur begrenzter pädagogischer Qualität ist, dürfte klar sein.

Trotz dieses Anspruchs auf Orientierungsrelevanz gilt für den Religionsunterricht offenbar nach wie vor, dass der prozentuale Anteil global orientierter Bildung gering ausfällt und darüber hinaus auch die strukturelle Dimension des Themas - geschweige denn die Frage individueller Handlungsstrategien - kaum im Blick ist. ${ }^{21}$

Häufig fehlte und fehlt offenkundig im Zusammenhang religiöser Bildung das Bewusstsein dafür, dass eine Annäherung an diese Weltrisiko-Themen die Aufmerksamkeit für die politisch-institutionellen Gegebenheiten sowie die zivilgesellschaftlichen Möglichkeiten notwendig mitbedingt. Eine sachkundige politikbezogene Expertise oder schon allein die Bereitschaft, sich intensiver mit den komplexen Hintergründen zu befassen und diese dann auch mit zu thematisieren, stellt sowohl auf Seiten der Religionslehrkräfte wie auch unter bildenden Pfarrerinnen und Pfarrern - gegenüber einer primär emotional ausgerichteten thematischen Annäherung - erfahrungsgemäß immer noch eine rühmliche Ausnahme dar.

Auch ist zu erleben, dass in entsprechenden rhetorischen Angeboten häufig entwe-

${ }^{20}$ Vgl. Rat der EKD (Hg.), Der Entwicklungsdienst der Kirche - ein Beitrag für Frieden und Gerechtigkeit in der Dritten Welt. Gütersloh 1973.

${ }^{21}$ Vgl. G. Orth, Art. Dritte Welt, Eine Welt, in: N. Mette/F. Rockers (Hg.), Lexikon der Religionspädagogik Bd. 1. Neukirchen-Vluyn 2001, Sp. 355-360. 
der in überborden optimistischer Weise von den Mitgestaltungs- und Veränderungsmöglichkeiten hinsichtlich globaler Prozesse oder umgekehrt dann in geradezu depressiver Weise von den Unabänderlichkeiten der Weltläufte die Rede ist. Die notwendige Mischung eines realistischen Idealismus geht dabei aber dann gerade häufig verloren. Zudem kann man fragen, ob gegenwärtig angesichts der gesellschaftlichen Tendenz einer Fokussierung auf den nationalen oder gar lokalen Nahbereich die (vermeintlich) ferner gelegen Probleme überhaupt noch stark in den Blickpunkt kommen sollen oder überhaupt gefragt sind.

Worin kann dann aber nun die Kernaufgabe religiöser Bildung hinsichtlich dieses thematisch hochkomplexen Gegenstandsfeldes bestehen?

\section{Der spezifische Beitrag religiöser Bildung in weltzivilgesellschaftlicher Perspektive}

Grundsätzlich gilt für die Frage nach diesem spezifischen Beitrag, dass religiöse Bildung sich der allgemeinpädagogischen Standards globalen Lernens überhaupt erst einmal bewusst sein muss: Um eine Sensibilität für globale Zusammenhänge im Sinn weltzivilgesellschaftlicher Bildung zu wecken, sollte sie sich gerade an diesen Standards orientieren. Diese lassen sich dahingehend näher bestimmen, dass sachliches Wissen und Kenntnis über globale Zusammenhänge und Konfliktlagen - etwa über Entwicklungspolitik und Umweltbildung, Friedens- und Menschenrechtserziehung, aber auch interkulturelle und interreligiöse Dimensionen - vermittelt und die Fähigkeit zur Einordnung der komplexen Sachverhalte vergrössert werden soll. ${ }^{22}$ Von dort her sollten Kinder und Jugendliche ein realistisches Handlungsrepertoire entwickeln können, das von Empathie für die Fernsten getragen ist und zugleich zur solidarischen Praxis motiviert. Ein solches global ausgerichtetes Lernen steht damit immer auch im Zielhorizont von Persönlichkeitsbildung und Sozialem Lernen, das kognitive, emotionale und soziale Komponenten mit beinhaltet. Es bedarf pädagogisch gesprochen jedenfalls sogleich immer auch sehr konkreter Orientierungen, wo und wie Kinder und Jugendliche in idealistischem Sinn, aber auch auf sehr realistische Weise zur positiven Einflussnahme auf weltzivilgesellschaftliche Verhältnisse beitragen können. Notwendig ist von dort aus auch die Einübung in eine bestimmte Haltung und Sensibilität für fern(st)e Verhältnisse und zugleich die Sensibilisierung für die Tatsache, dass diese Verhältnisse für das eigene Leben nicht bedeutungslos sind, weil beides unmittelbar miteinander verbunden und in erheblichem Sinn voneinander abhängig ist. Zugleich sollte eine solche Bildung angesichts der hochkomplexen und oftmals fernen Zusammenhänge aber auch grundlegende Kompetenzen „für den Umgang mit Unwissenheit, Ungewissheit, Unbegrenztheit und Fremdheit“23 vermitteln.

\footnotetext{
${ }^{22}$ Vgl. I. Juchler, Weltgesellschaft als Herausforderung für die politische Bildung, in: W. Sander/A. Scheunpflug (Hg.), Politische Bildung in der Weltgesellschaft. Herausforderungen, Positionen, Kontroversen. Bonn 2011, 399-416.

${ }^{23}$ A.K. Treml, Globalisierung als pädagogische Herausforderung. Möglichkeiten und Grenzen einer weltbürgerlichen Erziehung, in: W. Sander/A. Scheunpflug (Hg.), Politische Bildung in der Weltgesellschaft. Herausforderungen, Positionen, Kontroversen. Bonn 2011, 190-203, hier 200.
} 
Fragt man dabei nach dem spezifischen Beitrag religiöser Bildung zur Kommunikation und Deutung der weltzivilgesellschaftlichen Dynamiken, so soll damit keineswegs einer Politisierung das Wort geredet werden, bei der dann eine bestimmte religiöse Semantik lediglich zur Legitimierung oder gar Kaschierung einer schon bestehenden festen politischen Weltsicht dienen würde. Vielmehr geht es im Sinn einer öffentlichen Religionspädagogik - mindestens in ihrer christlichen Verankerung - entscheidend um die Frage, welche Perspektiven eine religiös profilierte Wahrnehmung der Dynamiken dieser Weltverhältnisse für ein besseres Verstehen und Handeln - als Beitrag zu einer demokratischen Allgemeinbildung im Licht des Evangeliums - eröffnen kann. ${ }^{24}$ Dass gerade zwischen den religiös-institutionellen Sozialformen des Christentums und den Strukturen einer Weltzivilgesellschaft große Affinitäten bestehen, macht M.L. Stackhouse deutlich, wenn er für das katholische „hierarchisch-subsidiäre“ wie für das spezifisch reformierte „föderal-bundesorientierte“ Modell formuliert, dass beide Modelle die Gefahren eines libertinistischen Individualismus ebenso vermeiden wie die eines politischen Kollektivismus: „both support the view that between the persons and the collective are the decisive, differentiated mediating institutions that sustain life, the fabric of civil society“. ${ }^{25}$

Es ließen sich hier nun, um einmal beim jüdisch-christlichen Überlieferungsgut zu bleiben, eine ganze Reihe von wertbezogenen biblischen Axiomen und Motiven benennen, die sich zur kritischen Deutung der globalen Entwicklungen und des „Mitwebens" am weltzivilgesellschaftlichen Stoff anbieten ${ }^{26}$ :

Gleichwohl sei im Folgenden lediglich nur auf zwei Aspekte kurz zu sprechen gekommen:

Zum einen stellt die biblische Überlieferung der besonderen Aufmerksamkeit auf die Entrechteten und Armen eine wesentliche Komponente für ein gegenwärtiges Weltverstehen dar. Es scheint mir allerdings infolge der aktuellen Weltverhältnisse sinnvoller, hier nicht von einer Option für die Armen zu sprechen, da dies zu stark als auf materielle Armut fokussiert erscheint. Angemessener erscheint die Rede von einer Option für die Schwachen, da dadurch die Vielfalt gegenwärtiger Herausforderungen sehr viel eindrücklicher und umfassender zum Ausdruck kommen kann und dabei der Aufmerksamkeitshorizont hin zur Frage erheblich erweitert wird, was den Menschen in seiner Würde unbedingt ausmacht. Die gegenwärtig viel beschworenen Bildungsaspekte der Teilhabegerechtigkeit und Bildungsgerechtigkeit sind somit gerade auch für eine weltzivilgesellschaftliche religiöse Bildung unbedingt mit zu berücksichtigen.

Zum anderen stellt die Hoffnung auf einen „ewigen Frieden“ und auf eine sich endgültig durchsetzende und manifestierende Gerechtigkeit gleichsam die biblisch

${ }^{24}$ Vgl. dazu jetzt W.F. Storrar/P.J. Casarella/P.L. Metzger (Eds.), A World for all? Global Civil Society in Political Theory and Trinitarian Theology. Grand Rapids 2011.

${ }^{25}$ M.L. Stackhouse, Civil Society, Religion, and the Ethical Shape of Polity, in: W.F. Storrar/P.J. Casarella/ P.L. Metzger (Eds.), A World for all? Global Civil Society in Political Theory and Trinitarian Theology. Grand Rapids 2011, 73.

${ }^{26}$ Vgl. G. Theißen, Zur Bibel motivieren. Aufgaben, Inhalte und Methoden einer offenen Bibeldidaktik. Gütersloh 2003. 
Grundvision überhaupt dar - wie sich dies etwa in entsprechenden ökumenischen Schwerpunktsetzungen etwa der Friedensdekade oder diverser Erlassjahr-Kampagnen auch immer wieder manifestiert hat.

In diesem Sinn ist im Kontext einer weltzivilgesellschaftlichen religiösen Bildung die paulinische Rede vom Bürgerrecht im Himmel (Phil 3,20) nicht als kontrafaktische Botschaft zu lesen, sondern angesichts der Rettungstat Jesu Christi gerade als Ermutigung zur Um- und Neugestaltung der hiesigen Weltverhältnisse: Diese eschatologischhoffnungsvolle Ankündigung inmitten der „Situation der Christen in der Welt“27 eröffnet jedenfalls die Perspektive, schon hier und jetzt „Protestleute gegen den Tod“ (Kurt Marti) zu werden.

Ganz grundsätzlich sollte gerade die spezifische jüdisch-christliche Glaubensgeschichte eine erhebliche Sensibilität für Leiden und Ungerechtigkeit gegenüber anderen religiösen Überzeugungen mit sich bringen und erst recht den Blick auf die leidvollen Ereignisse an ganz anderen - nur vermeintlich fremden - Orten der Weltzivilgesellschaft richten lassen. Das Bewusstsein, zu Gottes Schöpfung zu gehören, muss mit der Einsicht einhergehen, auch mitverantwortlich für das grössere Ganze der realen Weltverhältnisse zu sein. ${ }^{28}$

Ziel einer solchen kompetenzorientierten weltzivilgesellschaftlichen Bildung kann es dabei nun aber nicht sein, etwa im Sinn einer Bezugnahme auf ein eher allgemeines Weltethos oder die Hoffnung auf eine globale Zivilreligion alle faktischen Religionsprofile und -traditionen einfach außer Acht zu lassen oder gar negieren zu wollen. Religiöse Bildung zur weltzivilgesellschaftlichen Mitgestaltung sollte insofern eher vom Ziel einer zivilisierten und profiliert zivilisierenden Pluralität der Religionen ausgehen. ${ }^{29}$

Ein zivilisierender Religionspluralismus ist dabei auf eine möglichst friedliche Auseinandersetzung mit den unterschiedlichen religiösen Wahrheitsansprüchen notwendigerweise angewiesen. ${ }^{30}$ Insofern kann religiöse Bildung gerade angesichts der weltreligiösen Herausforderungen nur noch unter Berücksichtigung seiner ökumenischen und interreligiösen Dimension gedacht und konzipiert werden. ${ }^{31}$ Das ökumenische Lernen selbst ist von dort her nochmals neu auf seine interreligiöse Dimension im weltweiten Maßstab hin zu durchdenken. Vor dem aufgezeigten jüdisch-christlichem Erfahrungsund Glaubenshorizont lassen sich die Freiheits- und Verantwortungsspielräume innerhalb der Weltzivilgesellschaft prägnant reformulieren und in konkrete Bildungsprozesse ausarbeiten.

\footnotetext{
${ }^{27}$ F. Hahn, Theologie des Neuen Testaments. Bd. II: Die Einheit des Neuen Testaments. Tübingen ${ }^{3} 2011,490$.

${ }^{28}$ Vgl. zur systematischen Grundlegung nun den instruktiven Band von F.F. Fiorenza/K. Tanner/M. Welker (Hg.), Politische Theologie. Neuere Geschichte und Potenziale. Neukirchen-Vluyn 2011.

29 Vgl. K.E. Nipkow, Ziele interreligiösen Lernens als mehrdimensionales Problem, in: P. Schreiner/U. Sieg/ V. Elsenbast (Hg.), Handbuch interreligiöses Lernen. Gütersloh 2005, 362-380.

30 Vgl. T. Schlag, Ist Wahrheit im Religionsunterricht sinnvollerweise kommunizierbar? Didaktische Reflexionen in interreligiöser Perspektive, in ZPT 64 (2012), 46-55.

${ }^{31}$ Vgl. S. Fritsch-Oppermann, Globalisierung als Bedingung interreligiösen Lernens, in: P. Schreiner/U. Sieg/ V. Elsenbast (Hg.), Handbuch interreligiöses Lernen. Gütersloh 2005, 18-27.
} 


\section{Wie man in weltzivilgesellschaftlicher Perspektive von einer religiösen Grundhaltung und Überzeugung aus politisch und zivilgesellschaftlich bilden kann}

Gerade angesichts der gegenwärtigen globalen Verhältnisse hat die klassische religiöse Bildung am Ort der Schule ebenso wie in den verschiedenen kirchlichen Kontexten neu über ihre - im wahrsten Sinn des Wortes gegebene - Verortung nachzudenken. In diesem Zusammenhang kann der Grundgedanke von Schule und Kirche als intermediären Institutionen von weiterführender Bedeutung sein: Die zivilgesellschaftliche Vermittlungsaufgabe besteht dann etwa darin, bestimmte politische Fragen mit den individualistischen Vorstellungen von Gerechtigkeit auf die Ebene eines gemeinsamen Bildungsdiskurses zu bringen. Kirchlich verortete Bildungsträger können sich dabei bewusst am Selbstverständnis von Kirche als einer „public church“ und der damit verbundenen "public theology“ ausrichten. ${ }^{32}$

Konkret bieten sich gerade angesichts der zivilgesellschaftlichen Dynamiken Möglichkeiten der Vernetzung mit außerschulischen Trägern und Einrichtungen, die etwa konkrete Projektarbeit wie z. B. den Einsatz in bestimmten religiös und kirchlich orientierten weltzivilgesellschaftlichen NGO's mit sich bringen können. Wie etwa Erfahrungsberichte in Schülerzeitschriften über freiwillige Dienste von ehemaligen Schülern zeigen, können hier grundsätzlich sehr engere Verbindungen zwischen schulischem Lernen und erfahrungsorientiertem Lernen initiiert werden. Durch solche Praxisformen bis hin zur damit verbundenen Anschaulichkeit, Exemplarizität und Narrativität kann gerade diese globale Dimension religiöser Bildung anschaulich werden und nachhaltig wirksam sein. Der Religionsunterricht sollte sich hier gerade als ein wegbereitendes Fach für die Motivierung zu bzw. die Information über solche weltzivilgesellschaftlichen Engagementmöglichkeiten verstehen.

Aber auch im Fall der klassisch kirchlichen Bildungspraxis etwa der Konfirmationsund Jugendarbeit bestehen erhebliche Gelegenheiten für die Schaffung eines solchen weltzivilgesellschaftlichen Bewusstseins, etwa durch die bewusste Ausweitung des Themenspektrums auf globale Zusammenhänge hin, die sich am Ende eben nicht nur in einer Spende für „Brot für die Welt“ manifestieren, sondern durch die eine dezidiert politische und öffentliche Projektarbeit befördert wird. Dass dabei das anfangs erwähnte breite Begriffsspektrum von seinem analytisch-deskriptiven bis hin zum normativ-idealistischen Gebrauch mindestens mit in den Blick kommen muss, versteht sich von selbst.

Wenn sich, wie oben ausgeführt, Weltzivilgesellschaft durch Phänomene einer Weltkommunikation auszeichnet, müssen auch im Zusammenhang religiöser Bildung diese institutionell verorteten als persönlich bedeutsame Kommunikationsvorgänge gepflegt und eingeübt werden. Konkret gesprochen ist eine zivilisierte und zivilisierende religi-

\footnotetext{
${ }^{32}$ Vgl. dazu exemplarisch H. Bedford-Strohm, Poverty and Public Theology: Advocacy of the Church in Pluralistic Society, in: International Journal of Public Theology 2 (2008), 144-162 und zum internationalen Diskussionsstand neben dem International Journal of Public Theology auch W.F. Storrar/A.R. Morton (Eds.), Public Theology für the 21st Century. Essays in honour of Duncan B. Forrester. London/New York 2004.
} 
öse Kommunikation unbedingt zu fördern, die den Sprachlosen und Unbeachteten wieder eine Stimme und Aufmerksamkeit gibt und auf diese Weise auch neue kommunikative Beziehungen initiiert - bis hin zu einer Gedächtniskultur derer, die durch den eigenen Tod aus den kommunikativen Beziehungen endgültig ausgeschlossen zu werden drohen. Insofern hat eine weltzivilgesellschaftlich orientierte Kommunikation immer auch das Element des Nicht-Vergessen-Dürfens mit im Blick.

Zugleich muss sich die theologische Kommunikation in den je einzelnen Bildungsprozessen selbst an den globalen Sachverhalten auf ihre individuelle Relevanz hin überprüfen lassen und immer wieder selbst bewähren. Gerade für Kinder und Jugendliche lebt die Plausibilität religiöser Bildung entscheidend davon, dass die biblischen Visionen einer besseren, gerechteren Welt mit konkreten Handlungsmöglichkeiten, also der Logos biblischer Überlieferung mit dem politikrelevanten Ethos stimmig verbunden werden können. ${ }^{33}$

\section{Zurück zum "frustrierenden" Anfang}

Kehren wir noch einmal an die existenziellen und zugleich frustrierenden Erfahrungen der Jugendlichen zurück, von denen anfangs die Rede war. Dass sich ein dezidiert evangelischer Bildungsort wie der einer Evangelischen Akademie eines solchen Themas annimmt, spricht für die Ausrichtung dieses Bildungsortes selbst. Gleichwohl ist natürlich immer auch selbstkritisch zu fragen, ob religiöse Bildung am Ende eben doch nicht mehr als eine Spielwiese für die Sicheren und Etablierten darstellt? Bleiben solche Planspiele nicht Laborexperimente, in denen letztlich auf scheinheilige Weise bestimmte Emotionen erzeugt werden, ohne dass dabei aber wirklich Entscheidendes ausgelöst wird? Bleibt dies nicht gleichsam das pädagogische Äquivalent zu einer hoffnungslosen Symbolpolitik? Und weiter gefragt: Kann und sollte insbesondere die christliche Religion etwa in den Konflikten inmitten der arabischen Welt überhaupt Position beziehen und wenn ja, in welcher Richtung?

Das Ergebnis mag, wie im geschilderten Fall, Frustration und Ratlosigkeit ausgelöst haben. Zugleich erschließt sich gerade durch eine solche Erfahrung eine wichtige Grunderkenntnis: Die Verhältnisse im Weltmaßstab sind komplex und herausfordernd, aber zugleich auch unmittelbar fordernd und deutungsnotwendig - schon dies allein ist eine wesentliche Einsicht weltzivilgesellschaftlicher Bildung. So ermöglicht diese im Sinn von Frustrationstoleranz und Differenzierungskompetenz hoffentlich auch konstruktive Erfahrungen. Im besten Fall wird die Sensibilität und auch die Fähigkeit erhöht, mit den unlösbaren Konflikten der eigenen Gegenwart so umzugehen, dass deren zerstörerisches und selbstzerstörerisches Potenzial wenigstens wahrgenommen und sich im Rahmen der individuellen Möglichkeiten damit auseinandergesetzt wird.

${ }_{33}$ Vgl. T. Schlag/F. Schweitzer, Brauchen Jugendliche Theologie? Jugendtheologie als Herausforderung und didaktische Perspektive. Neukirchen-Vluyn 2011. 
Und dies immer in Erinnerung daran, „that the most important question to ask about religion is not wether it is useful but wether it is true". ${ }^{34}$

Dazu braucht es nicht mehr, aber auch nicht weniger, als den christlichen Glauben als Religion der Freiheit und Verantwortung zu verdeutlichen und von dort aus Kinder und Jugendliche dazu zu ermutigen, zu freien und verantwortlichen Akteuren inmitten der Weltzivilgesellschaft zu werden. Eine solche zivile, zivilisierte und zivilisierende religiöse Bildung, die sich sowohl die Vision gelingenden weltlichen wie himmlischen Bürgerrechts erhält, kann dann im wahrsten Sinn des Wortes Welt erschließende sinnstiftende Bedeutung erlangen.

Michael Haspel

\section{Gottebenbildlichkeit und Menschenwürde - Implikationen für Bildung und öffentlichen Diskurs in Martin Luther King, Jr.'s Konzeption „Öffentlicher Theologie"}

Zunächst wurde Martin Luther King, Jr. vor allem als Bürgerrechtsaktivist wahrgenommen, zum einen quasi hagiografisch, zum anderen aber auch in kritischer Ablehnung etwa durch den radikaleren Flügel der Bürgerrechtsbewegung. Erst in darauf folgenden Phasen sind seine Rolle als öffentlicher Redner und, mit einiger zeitlicher Verzögerung, vor allem im angelsächsischen Bereich sein eigenständiger Beitrag als Theologe in den Fokus der Aufmerksamkeit gerückt. ${ }^{1}$ Verschiedentlich wurde inzwischen auf den Aspekt der public education bei King verwiesen. In diesem Artikel möchte ich diesem Hinweis auf Kings implizite Bildungskonzeption nachgehen und dazu die Konsequenzen von Kings grundlegendem theologischen Verständnis der Gottebenbildlichkeit für das in seiner „Öffentlichen Theologie“ beinhaltete theologische Bildungsverständnis und die Konsequenzen für verständigungsorientierte, öffentliche Diskurse untersuchen. Dazu rekonstruiere ich zunächst Kings theologische Begründungen einer „Öffentlichen Theologie“ anhand seiner eigenen Texte (1), und unternehme dann eine vorläufige Verhältnisbestimmung von „Öffentlicher Theologie“ und public education in Auseinandersetzung mit der einschlägigen Analyse von Downing (2). Daran anknüpfend können anhand von Wills’ Analyse von Kings Verständnis der Gottebenbildlichkeit die Konsequenzen für Bildung und öffentlichen Diskurs dann

\footnotetext{
${ }^{34}$ P. L. Berger, Religion and Global Civil Society, in: M. Juergensmeyer (Ed.), Religion in Global Civil Society, Oxford 2005, 11-22, hier 21.

${ }^{1}$ Dieser Forschungsstand ist manifestiert in der Sammlung der damaligen Aufsatzliteratur bei David Garrow (ed.), Martin Luther King, Jr. Civil Rights Leader, Theologian, Orator. Vol. I-III. Brooklyn/New York 1989.
} 